Gut and Liver, Vol. 11, No. 1, January 2017, pp. 38-46

REVIEW

\title{
CpG Island Methylator Phenotype-High Colorectal Cancers and Their Prognostic Implications and Relationships with the Serrated Neoplasia Pathway
}

Ye-Young Rhee, Kyung-Ju Kim, and Gyeong Hoon Kang

Department of Pathology, Seoul National University College of Medicine, Seoul, Korea

The concept of a $\mathrm{CpG}$ island methylator phenotype (CIMP) was first introduced by Toyota and Issa to describe a subset of colorectal cancers (CRCs) with concurrent hypermethylation of multiple $\mathrm{CpG}$ island loci. The concept of CIMP as a molecular carcinogenesis mechanism was consolidated by the identification of the serrated neoplasia pathway, in which CIMP participates in the initiation and progression of serrated adenomas. Distinct clinicopathological and molecular features of CIMP-high (CIMP-H) CRCs have been characterized, including proximal colon location, older age of onset, female preponderance, and frequent associations of high-level microsatellite instability and BRAF mutations. CIMP-H CRCs arise in sessile or traditional serrated adenomas and thus tend to display the morphological characteristics of serrated adenomas, including epithelial serration, vesicular nuclei, and abundant cytoplasm. Both the frequent association of $\mathrm{CIMP}$ and poor prognosis and different responses of CRCs to adjuvant therapy depending on CIMP status indicate clinical implications. In this review, we present an overview of the literature documenting the relevant findings of CIMP-H CRCs and their relationships with the serrated neoplasia pathway. (Gut Liver 2017;11:38-46)

Key Words: Colon cancer; Colorectal cancer; $\mathrm{CpG}$ island methylator henotype; Microsatellite instability; Serrated neoplasia

\section{INTRODUCTION}

In the human genome, methylation occurs at the fifth carbon of a cytosine in the context of a CpG dinucleotide and 5-methylcytosine (5-mC) accounts for approximately $4 \%$ of the cytosine in the human genome. In normal cells, CpG sites located in the promoter $\mathrm{CpG}$ island loci are protected from methylation, whereas those located in genomic sequences other than the promoter $\mathrm{CpG}$ island loci tend to be methylated. In particular, CpG sites of repetitive DNA elements are heavily methylated. In cancer cells, CpG sites undergo methylation changes in opposite directions depending on their location in the genome. $\mathrm{CpG}$ sites, which are protected from DNA methylation in normal cells, tend to undergo hypermethylation, whereas CpG sites that are heavily methylated in normal cells tend to undergo demethylation. Thus, DNA methylation changes that occur in cancer cells are characterized by both focal promoter CpG island hypermethylation and diffuse genomic hypomethylation. However, there is no physical link between promoter $\mathrm{CpG}$ island hypermethylation and genomic hypomethylation. Although promoter $\mathrm{CpG}$ island hypermethylation is closely associated with inactive status of gene promoter activity, cancer-associated hypermethylation usually involves inactive genes that are basally or not expressed in normal cells. For colorectal cancers (CRCs), of the genes that show cancer-associated hypermethylation in their promoter CpG island loci, approximately 10\% are active genes that are expressed in normal colonic epithelial cells. ${ }^{1,2}$ Inactivation of actively expressed tumor suppressor genes or tumor-related genes by promoter $\mathrm{CpG}$ island hypermethylation contributes to tumorigenesis by leading to cell proliferation or inhibiting cell apoptosis or senescence.

Although promoter $\mathrm{CpG}$ island hypermethylation is found in virtually every tissue type of human cancer, a subset of CRCs show concordant hypermethylation of numerous gene promoter $\mathrm{CpG}$ island loci. The concept of a CpG island methylator phenotype (CIMP) was introduced to refer to such a subset of CRCs with widespread hypermethylation of numerous promoter CpG island loci. Akin to microsatellite instability (MSI), CIMP is now recognized as one of the most important molecular car-

\footnotetext{
Correspondence to: Gyeong Hoon Kang

Department of Pathology, Seoul National University College of Medicine, 103 Daehak-ro, Jongno-gu, Seoul 03080, Korea

Tel: +82-2-740-8263, Fax: +82-2-743-5530, E-mail: ghkang@snu.ac.kr

Received on October 23, 2015. Accepted on March 29, 2016. Published online November 28, 2016

pISSN 1976-2283 eISSN 2005-1212 https://doi.org/10.5009/gnl15535

@ This is an Open Access article distributed under the terms of the Creative Commons Attribution Non-Commercial License (http://creativecommons.org/licenses/by-nc/4.0) which permits unrestricted non-commercial use, distribution, and reproduction in any medium, provided the original work is properly cited.
} 
cinogenesis pathways of CRCs, and CIMP-high (CIMP-H) CRCs have been characterized for their clinicopathological features. Furthermore, their premalignant lesions have been identified to be sessile serrated adenomas and traditional serrated adenomas. The serrated neoplasia pathway, another morphological multistep pathway parallel to the classical adenoma-carcinoma sequence pathway, has been proposed to describe multistep progression from sessile or traditional serrated adenomas to adenocarcinomas. CIMP-H CRCs are now understood to develop along the serrated neoplasia pathway. In this review, we present an overview of the literature documenting the clinicopathological and molecular features of CIMP-H CRCs, the candidate causes of CIMP, the relationship between CIMP-H CRCs and serrated pathway adenocarcinomas, the diagnostic marker panels of CIMP, the prognostic implications of CIMP status, and the clinicopathological features of serrated adenocarcinomas with an aim of consolidating our understandings about CIMP and serrated neoplasia pathways.

\section{CIMP AND SERRATED NEOPLASIA PATHWAYS}

CRC is a collection of heterogeneous diseases with not only molecular but also morphological aspects. Three molecular pathways are recognized in colorectal carcinogenesis, including chromosomal instability (CIN), MSI, and CIMP pathways. The CIN pathway is characterized by alterations in the number and structure of chromosomes, whereas the MSI pathway is characterized by alterations in the number of mono- or dinucleotide repeats in coding or regulatory sequences. CRCs develop through two different morphological multistep pathways, including the classical adenoma-carcinoma sequence and the serrated neoplasia pathway. ${ }^{3,4}$ The classical adenoma-carcinoma sequence refers to the concept that conventional adenomas, including tubular adenomas, tubulovillous adenomas, or villous adenomas, progress and transform into invasive adenocarcinomas. The serrated neoplasia pathway is a term describing the notion that sessile serrated adenomas or traditional serrated adenomas progress into invasive adenocarcinomas. ${ }^{5}$ The CIN pathway is the underlying molecular mechanism for the classical adenoma-carcinoma sequence, although hereditary MSIhigh (MSI-H) tumors known as Lynch syndrome, develop along the classical adenoma-carcinoma sequence. Premalignant lesions of Lynch syndrome are manifested as tubular adenomas with varying villosity. In contrast with Lynch syndrome tumors, the vast majority of sporadic MSI-H CRCs are thought to arise in CIMP-H sessile serrated adenomas with BRAF mutation, through methylation-associated inactivation of MLH1..$^{6-8}$ Thus, MSI-H tumors follow different morphological multistep routes depending on their causes: hereditary and sporadic MSI-H CRCs follow the classical adenoma-carcinoma sequence and serrated neoplasia pathway, respectively. ${ }^{9-12}$ In contrast with CIMP-H/ MSI-H CRCs, CIMP-H/non-MSI-H CRCs are thought to develop from pre-existing traditional serrated adenomas. ${ }^{13}$ In general, the CIN pathway accounts for 65\% to 75\% of CRCs, whereas the CIMP pathway and hereditary MSI pathway account for 20\% to $30 \%$ and 5\% of CRCs, respectively. ${ }^{14,15}$ However, the proportions of molecular carcinogenesis pathways differ depending on geographical areas. In Korean people, the CIMP pathway accounts for approximately $10 \%$ of CRCs. ${ }^{16,17}$ Even within the same geographical areas, the proportions of molecular carcinogenesis pathways differ among races. In the United States, the proportion of CIMP pathways is higher in Caucasians than in AfricanAmericans or Asian-Americans. ${ }^{18}$ Regardless of geographical areas or race, the proportion of the CIMP pathway is higher in females than in males, in older people than in younger people, and in proximal bowel subsites than in distal bowel or rectum subsites. ${ }^{18-24}$

\section{CAUSE OF CIMP IN CRCS AND THEIR PREMALIGNANT LESIONS}

DNA methylation is mediated by DNA methyltransferases, including DNMT1, DNMT3A, and DNMT3B. DNMT3A and DN$M T 3 B$ are de novo methylation enzymes, whereas DNMT1 is a maintenance methylation enzyme. Mutations of these DNA methyltransferase genes are found in CRCs at frequencies $<8 \%$ and mainly in MSI-H CRCs (http://www.cbioportal.org) but not in CIMP-H/non-MSI-H CRCs, which indicates that DNMT gene mutations are secondary to mismatch repair defects. DNMT gene mutations cannot be a cause for CIMP-H because CIMP-H appears prior to MSI-H in sessile serrated adenomas. Amplification of DNMT3B is found in $9 \%$ to $14 \%$ of CRCs but is not observed in CIMP-H or MSI-H CRCs. Ten-eleven translocation (TET) enzymes TET1, TET2, and TET3 are DNA demethylases, which are capable of oxidizing the methyl group of $5-\mathrm{mC}$ and thus converting 5-mC into 5-hydroxymethyl cytosine. Mutations of TET genes, which are found in CRCs at frequencies $<8 \%$, are mainly found in MSI-H CRCs and rarely found in CIMP-H/non-MSI-H CRCs. Data from the Cancer Genome Atlas (TCGA) indicates no associations between genetic alterations of DNA methyltransferase or demethylase genes and CIMP-H CRCs. Isocitrate dehydrogenase (IDH) enzymes IDH1 and IDH2 catalyze the oxidative decarboxylation of isocitrate, producing $\alpha$-ketoglutarate. Gain of function mutations in $I D H 1$ or $I D H 2$ enable mutant IDH1 or IDH2 enzymes to produce 2-hydoxyglutarate, which inactivates $\alpha$-ketoglutarate-dependent dioxygenases, including KDM4A and KDM4C, H3K9/H3K36 demethylases, and TET2. However, mutations of $I D H 1$ and $I D H 2$ are found in $\leq 2 \%$ of CRCs, mainly in MSI-H CRCs. IDH1 and IDH2 mutations are not found in CIMP-H/non-MSI-H CRCs (http://www.cbioportal.org). ${ }^{25}$ These findings suggest that mutations of IDH1 and IDH2 are secondary to MSI and do not cause CIMP in non-MSI-H CRCs.

Viral infection may cause aberrant DNA methylation, which is evidenced by Epstein-Barr virus (EBV)-positive gastric can- 
cers. ${ }^{26}$ For CRCs, EBV infection is detected in $19 \%$ to $52 \%$ of CRC tissue specimens by polymerase chain reaction (PCR). ${ }^{27,28}$ However, in situ hybridization analyses for the detection of EBER1-2 RNAs demonstrated EBVs in infiltrating nonneoplastic lymphoid cells but not in tumor cells, which contrasts with EBV-positive gastric cancers in which EBV in situ hybridization exhibit EBVs in nearly all of the tumor epithelial cells. ${ }^{26,27}$ Regardless of whether EBV is present in tumor cells or not, there is no relationship between the presence of EBV in cancer tissue specimens and CIMP. ${ }^{28}$ Although there are controversies concerning JCV infection, which was found in $0 \%$ to $86 \%$ of the tumor samples tested in the literature, ${ }^{29-31}$ Goel et al. ${ }^{32}$ demonstrated an association between John Cunningham virus (JCV) Tantigen expression in cancer cells and promoter $\mathrm{CpG}$ island hypermethylation of multiple genes (MLH1, CDKN2A [p16], PTEN, and RUNX3), and further suggested that JCV T-antigen might induce CIMP. However, such a link between expression of JCV T-antigen and promoter $\mathrm{CpG}$ island hypermethylation has not been reproduced in other studies. ${ }^{32}$

The strong association between BRAF mutation and CIMP-H CRCs has led many researchers to postulate that BRAF mutation might be involved in the initiation of CIMP. Rad et al. ${ }^{33}$ developed $B R A F$ knockin mice to study the role of $B R A F^{\mathrm{V} 600 \mathrm{E}}$ in intestinal tumorigenesis and have demonstrated that $B R A F^{\mathrm{V} 600 \mathrm{E}}$ initiates the serrated neoplasia pathway in which serrated lesions progressed from hyperplastic polyps to adenocarcinomas through traditional serrated adenomas. How BRAF mutation leads to aberrant promoter $\mathrm{CpG}$ island hypermethylation and generation of CIMP remained unclear until Fang et al. ${ }^{34}$ demonstrated that $B R A F$ mutant proteins directly induce promoter
CpG island methylation by selectively promoting the assembly of the MAFG corepressor complex in CIMP target genes. However, there has been no additional confirmatory study reported to support this finding.

Because dietary factors such as folate, methionine, and alcohol are involved in DNA methylation processes, diets low in folate and methionine and high consumption of alcohol have been hypothesized to be associated with an increased risk of CRCs with CpG island methylation. ${ }^{35,36}$ However, the current literature does not support the effect of dietary factors in the increased likelihood of CIMP-H colon cancers. ${ }^{18}$ Instead, smoking has been demonstrated to be associated with an increased risk of CIMP-H CRCs. ${ }^{37}$ In a recent study of Weisenberger et al. ${ }^{38}$ who analyzed the CIMP status of 3,119 population-based CRCs from the multinational colon cancer family registry and assessed etiologic heterogeneity in a case-case study, the association between smoking and CIMP was valid only for female CRC patients.

\section{DIAGNOSTIC MARKER PANELS OF CIMP}

In 1999, the Issa team found that CRCs were bimodally distributed in terms of the number of methylated $\mathrm{CpG}$ island loci that were methylated in a cancer-specific manner (Table 1). They analyzed CRCs for their methylation statuses in seven cancer-associated methylation markers (MINT1, MINT2, MINT12, MINT17, MINT25, MINT27, and MINT31) using methylated $\mathrm{CpG}$ island amplification and considered a tumor CIMP-positive (CIMP-H) if the tumor showed methylation in at least three methylation markers. ${ }^{39}$ In their study, approximately 50\% of

Table 1. Diagnostic Marker Panels of the CIMP and Cutoff Values for CIMP-H Tumors

\begin{tabular}{|c|c|c|c|c|}
\hline Author (year) & $\begin{array}{l}\text { Methylation analysis } \\
\text { methodology }\end{array}$ & Panel markers & $\begin{array}{l}\text { Cutoff values } \\
\text { for CIMP-H }\end{array}$ & $\begin{array}{l}\text { Percentage of CIMP-H tumors (total number } \\
\text { of study cases, tissue sample type) }\end{array}$ \\
\hline Toyota et al. (1999) ${ }^{39}$ & COBRA & $\begin{array}{l}\text { MINT1, MINT2, MINT12, MINT17, } \\
\text { MINT25, MINT27, and MINT31 }\end{array}$ & $\geq 3 / 7$ & $50 \%$ of CRCs ( $n=43$, fresh tissue sample) \\
\hline Samowitz et al. $(2005)^{42}$ & MSP & $\begin{array}{l}\text { MINT1, MINT2, MINT31, } \\
\text { CDKN2A (p16), and MLH1 }\end{array}$ & $\geq 2 / 5$ & $\begin{array}{l}30 \% \text { of colon cancers ( } \mathrm{n}=864 \text {, FFPE tissue } \\
\text { sample) }\end{array}$ \\
\hline $\begin{array}{l}\text { Weisenberger et al. } \\
(2006)^{23}\end{array}$ & MethyLight & $\begin{array}{l}\text { CACNA1G, IGF2, NEUROG1, } \\
\text { RUNX3, and SOCS1 }\end{array}$ & $\geq 3 / 5$ & $18 \%$ of CRCs ( $\mathrm{n}=187$, fresh tissue sample) \\
\hline Ogino et al. $(2006)^{57}$ & MethyLight & $\begin{array}{l}\text { CACNA1G, CDKN2A (p16), } \\
\text { CRABP1, MLH1, and NEUROG1 }\end{array}$ & $\geq 4 / 5$ & $17 \%$ of CRCs ( $n=460$, FFPE tissue sample) \\
\hline Ogino et al. $(2007)^{24}$ & MethyLight & $\begin{array}{l}\text { CACNA1G, CDKN2A (p16), } \\
\text { CRABP1, IGF2, MLH1, NEUROG1, } \\
\text { RUNX3, and SOCS1 }\end{array}$ & $\geq 6 / 8$ & $15 \%$ of CRCs ( $n=920$, FFPE tissue sample) \\
\hline Kim et al. (2009) $)^{43}$ & MethyLight & $\begin{array}{l}\text { CACNA1G, CDKN2A (p16), } \\
\text { CRABP1, IGF2, MLH1, NEUROG1, } \\
\text { RUNX3, and SOCS1 }\end{array}$ & $\geq 5 / 8$ & $12 \%$ of CRCs ( $n=320$, FFPE tissue sample) \\
\hline
\end{tabular}

CIMP, CpG island methylator phenotype; CIMP-H, CpG island methylator phenotype-high; COBRA, combined bisulfite restriction analysis; CRC, colorectal cancer; MSP, methylation-specific polymerase chain reaction; FFPE, formalin-fixed, paraffin-embedded. 
randomly selected CRC tissue samples $(n=43)$ were CIMP-H. Of the seven markers, MINT25 had low sensitivity and specificity for detection of CIMP and thus was excluded from the CIMP marker panel in a subsequent study in which they used combined bisulfite restriction analysis for the methylation analysis. ${ }^{40}$ The Issa team further changed the CIMP marker panel and used a reduced set of five markers, namely MINT1, MINT2, MINT31, CDKN2A (p16) and MLH1. ${ }^{41}$ Samowitz et al. ${ }^{42}$ used methylationspecific PCR (MSP) for the methylation analysis of the same five-marker panel and found that $30 \%$ of colon cancers $(n=864)$ were CIMP-H (methylated in two or more of the five markers). In 2006, the Laird team developed a new five-marker panel (CACNA1G, IGF2, NEUROG1, RUNX3 and SOCS1) through a systematic stepwise screening of $195 \mathrm{CpG}$ island loci using real-time PCR-based MethyLight assay. ${ }^{23}$ In their study, 18\% of CRCs $(n=187)$ showed methylation in three or more of the five markers and were defined as CIMP-H. In 2007, Ogino et al. ${ }^{24}$ proposed a MethyLight-based eight-marker panel (CACNA1G, CDKN2A [p16], CRABP1, IGF2, MLH1, NEUROG1, RUNX3, and SOCS1) that incorporated the Laird team's five-marker panel. In Ogino et al.'s study, ${ }^{24} 15 \%$ of CRCs ( $\left.n=920\right)$ were CIMP-H with $\geq 6 / 8$ methylated markers. In our previous study, a CIMP$H$ cutoff set at $\geq 5 / 8$ methylated markers was found to outperform a CIMP-H cutoff set at $\geq 6 / 8$ methylated markers because when compared with CIMP-H CRCs defined by $\geq 6 / 8$ methylated markers, CIMP-H CRCs defined by $\geq 5 / 8$ methylated markers showed stronger associations with known clinicopathological or molecular features of CIMP-H CRCs. ${ }^{43}$

CIMP status can be easily determined using the MethyLightbased methylation assays of Laird's five-marker panel or Ogino's eight-marker panel. For the five marker panel, tumors with methylation in $\geq 60 \%$ and $\leq 40 \%$ of the panel markers were defined as CIMP-H and CIMP-low (CIMP-L)/CIMP-0, respectively. In contrast with the five-marker panel, the eight-marker panel can detect tumors with methylation in 50\% of the panel markers, which might generate a dilemma as to whether such tumors with methylation in 50\% of the panel markers should be diagnosed as CIMP-H or CIMP-L/CIMP-0. Using the MethyLightbased methylation assay of the eight-marker panel, Bae et al. ${ }^{16}$ evaluated whether CRCs with methylation in 50\% of the panel markers are close to CRCs with methylation in $\leq 40 \%$ or $\geq 60 \%$ of the panel markers in terms of their clinicopathological and molecular features (accepted for publication). The findings indicate that CRCs with 50\% methylation of the panel markers are closer to those with methylation in $\geq 60 \%$ of the panel markers than those with methylation in $\leq 40 \%$ of the panel markers and that CRCs with 50\% of methylation of the panel markers should be classified as CIMP-H.

\section{CLINICOPATHOLOGICAL AND MOLECULAR FEATURES OF CIMP-HIGH CRCS WITH AN EMPHASIS ON KRAS/ BRAF MUTATIONS}

The original studies by the Issa team characterized CIMP-H CRCs as closely associated with proximal location, high level of MSI, KRAS mutation, and lack of TP53 mutation (Table 2). ${ }^{39,40}$ The relationship between CIMP and KRAS mutation or lack of TP53 mutation was independent of MSI status. The relationship between CIMP and BRAF mutation is not available in these original CIMP studies because the authors did not analyze $B R A F$ mutation. However, a later study by the Issa team revealed the relationship between CIMP and BRAF mutation. ${ }^{44}$ Nagasaka et

Table 2. Clinicopathological and Molecular Features of CIMP-H Colon Cancers or CRCs

\begin{tabular}{|c|c|c|}
\hline Author (year) & $\begin{array}{l}\text { Case number and } \\
\text { tissue sample type }\end{array}$ & Features of CIMP-H colon cancers or CRCs \\
\hline Toyota et al. $(1999)^{39}$ & 50 CRCs (fresh tissue) & Proximal location and frequent MSI-H \\
\hline Toyota et al. $(2000)^{40}$ & 88 CRCs (fresh tissue) & Frequent MSI-H, KRAS mutation, and lack of TP53 mutation \\
\hline Hawkins et al. (2002) ${ }^{49}$ & 426 CRCs (fresh tissue) & $\begin{array}{l}\text { Proximal location, female sex, older age, high tumor grade, mucinous type, } \\
\text { wild-type TP53, frequent MSI-H, and K-ras mutation }\end{array}$ \\
\hline Samowitz et al. $(2005)^{42}$ & 864 Colon cancers (FFPE) & Proximal colon location, older age, $B R A F$ mutation \\
\hline Weisenberger et al. (2006) ${ }^{23}$ & 187 CRCs (fresh tissue) & $\begin{array}{l}\text { Female, proximal location, frequent MSI-H, BRAF mutation, } \\
\text { low frequency of } K R A S \text { mutation }\end{array}$ \\
\hline Shen et al. $(2007)^{44}$ & 97 CRCs (fresh tissue) & $\begin{array}{l}\text { CIMP1 (proximal location and BRAF mutation) and } \\
\text { CIMP2 (proximal location and KRAS mutation) }\end{array}$ \\
\hline Simons et al. $(2013)^{47}$ & 509 CRCs (FFPE) & $\begin{array}{l}\text { CIMP-H/MSI-H (proximal location and BRAF mutation) and } \\
\text { CIMP-H/non-MSI-H (proximal location and KRAS mutation) }\end{array}$ \\
\hline The Cancer Genome Atlas (2012) ${ }^{46}$ & 212 CRCs (fresh tissue) & $\begin{array}{l}\text { CIMP-H/MSI-H (proximal location and BRAF mutation) and } \\
\text { CIMP-H/non-MSI-H (proximal location and KRAS mutation) }\end{array}$ \\
\hline
\end{tabular}

CIMP-H, CpG island methylator phenotype-high; CRCs, colorectal cancers; MSI-H, microsatellite instability-high; FFPE, formalin-fixed, paraffinembedded. 
al.'s study ${ }^{45}$ was the first to correlate BRAF mutation with CIMP. They analyzed 234 CRCs for their methylation statuses in 11 sites of $10 \mathrm{CpG}$ island loci and found that CRCs with BRAF mutations had significantly higher numbers of methylated markers than CRCs with KRAS mutations, whereas CRCs with KRAS mutations exhibited significantly higher numbers of methylated markers than CRCs with neither BRAF nor KRAS mutations. Because the clinicopathological features of CIMP-H CRCs overlap those of MSI-H CRCs, Samowitz et al. ${ }^{42}$ separated microsatellite stable colon cancers from microsatellite unstable colon cancers and then compared clinicopathological and molecular features between CIMP-H and non CIMP-H colon cancers. Samowitz et $a{ }^{42}$ analyzed five CpG island loci (MLH1, MINT1, MINT2, MINT31, and CDKN2A [p16]) using MSP and defined CIMP-H as methylation at 2 or more of these loci. Relationships between CIMP and proximal colon location, older age, or BRAF mutation were irrespective of MSI status. However, the frequency of a KRAS mutation was significantly lower in CIMP-H/MSI-H colon cancers than in non-CIMP-H/MSI-H colon cancers, which contrasted with the situation in microsatellite-stable colon cancers in which the KRAS mutation correlated with CIMP-H. The MethyLight-based five-marker panel (CACNA1G, NEUROG1, IGF2, RUNX3, and SOCS1) developed by the Laird team addressed a link between BRAF mutation and CIMP but no link between KRAS mutation and CIMP. ${ }^{23}$ In the Laird team's study, KRAS and BRAF mutations occurred in 10\% and 73\% of CIMPH CRCs ( $\mathrm{n}=33)$ and 35\% and 1\% in non-CIMP-H CRCs $(\mathrm{n}=154)$, respectively. CIMP-H/MSI-H and CIMP-H/non-MSI-H CRCs ( $\mathrm{n}=12$ vs $\mathrm{n}=21$ ) had KRAS mutations at frequencies of $0 \%$ and 16\%, respectively. Thus, CIMP-H CRCs defined by Laird team's CIMP marker panel were characterized by a low KRAS mutation frequency, which is irrespective of MSI status. By clustering the analysis of CRCs $(n=97)$ on the basis of a combination of genetic and epigenetic profiling, the Issa team identified three molecular subtypes of CRCs, including CIMP1 (23\%), CIMP2 (38\%), and CIMP-negative (39\%). ${ }^{44}$ The BRAF mutation was identified in 53\% of CIMP1, 3\% of CIMP2, and 19\% of CIMP-negative CRCs, whereas the KRAS mutation was found in 0\% of CIMP1, 100\% of CIMP2, and 19\% of CIMP-negative CRCs. A CRC study from the TCGA network identified four methylation clusters, namely CIMP-H, CIMP-L, cluster 3 and cluster 4, based on unsupervised clustering of the promoter DNA methylation profiles that were obtained using Illumina Infinium (HumanMethylation27) arrays. ${ }^{46}$ In a set of CRCs with sequencing and copy number alteration data ( $\mathrm{n}=212), 48 \%$ and 39\% of CIMP-H CRCs $(\mathrm{n}=31)$ had BRAF and KRAS mutations, respectively. The BRAF mutation was found in 65\% of CIMP-H/MSI-H CRCs $(n=20)$, whereas the KRAS mutation was identified in 73\% of CIMP-H/non-MSIH CRCs ( $\mathrm{n}=11)$. The BRAF and KRAS mutations were observed in $6 \%$ and $60 \%$ of CIMP-L CRCs ( $n=48)$, respectively. The TCGA data clearly indicates that the KRAS mutation is closely associated with CIMP-H in MSS CRCs, which is consistent with the findings of Samowitz et al.'s ${ }^{42}$ and Simons et al.'s ${ }^{47}$ studies.

\section{PROGNOSTIC OR PREDICTIVE IMPLICATIONS OF CIMP STATUS}

There have been controversies over the prognostic or predictive role of CIMP in colon cancer or CRC patients. The reasons for the inconsistency may include varying methodologies of methylation analysis, varying CIMP panel marker compositions, and uncontrolled case subjects with respect to cancer stage, adjuvant therapy, neoadjuvant therapy, gender, and bowel-subsite location of tumor. Studies that have evaluated the prognostic or predictive implications of CIMP status in colon cancers or CRCs could be divided into two major categories based on type of CIMP marker panels. Studies that included MINT CpG island loci in their CIMP panels could be categorized as "classic panel"based study, whereas studies that used Laird's five-marker panel or Ogino's eight-marker panel could be categorized as "new panel"-based study. "Classic panel"-based studies showed varying prognostic or predictive roles of CIMP in colon cancer or CRC patients. Only one study found better survival among CIMP-H CRCs compared with non-CIMP-H CRCs. Van Rijinsoever et $a{ }^{48}{ }^{4}$ investigated the predictive value of CIMP in stage III CRC patients (surgery alone $[n=150]$ vs adjuvant 5-fluorouracil/ leucovorin [FL; $n=150]$ ) by analyzing the methylation statuses of three methylation markers. CRCs with $\geq 2 / 3$ methylated markers, defined as CIMP-H, had better disease-free survival in the adjuvant setting versus the setting of surgery alone. The majority of "classic panel"-based studies demonstrated significant associations, in non-MSI-H CRCs, between worse survival and CIMP-H tumors. ${ }^{49-51}$ However, one study did not demonstrate an association between CIMP status and prognosis, even in nonMSI-H CRCs. ${ }^{42}$ Samowitz et al. ${ }^{42}$ evaluated a large populationbased sample of individuals with colon cancer $(n=816)$ and found that it was not CIMP-H ( $\geq 2 / 5$ methylated markers) but the BRAF mutation that was an independent prognostic marker of poor prognosis in non-MSI-H colon cancers.

"New panel"-based studies also described varying prognostic or predictive roles of CIMP in colon cancer or CRC patients. In one study, CIMP-H has been shown to be an independent predictor of better survival in colon cancer patients. ${ }^{52}$ Ogino et $a .^{52}$ analyzed the CIMP status of stage I to IV colon cancer patients ( $n=649$, from two prospective cohort studies) using the MethyLight-based eight-marker panel. In their study, CIMP-H was not a significant prognostic parameter in univariate analysis but was an independent predictor of better survival (a low colon cancer-specific mortality) in colon cancer patients after adjusting for MSI and BRAF mutation. ${ }^{52}$ By contrast, CIMP-H CRCs exhibited an increased risk of CRC-related death in Simons et al.'s study, ${ }^{47}$ which used Laird's five-marker panel and analyzed stage I to IV CRC patients ( $n=509$, from a prospective cohort study) to determine the association between CIMP status 
and survival. On the other hand, in Dahlin et al.'s study, ${ }^{53}$ which used Ogino's eight-marker panel, CIMP-H CRC patients had a significantly increased risk of cancer-specific death compared with CIMP-negative (CIMP-0) CRC patents in the setting of a non-MSI-H CRC group. However, the statistical significance was lost after adjusting the multivariate model for the presence of $B R A F$ mutations, which suggests that CIMP-H/non-MSI-H CRCs with $B R A F$ mutation might have a higher risk of cancer-specific death. Consistently, poorer survival for CIMP-H/non-MSI-H CRCs with BRAF mutation has been highlighted in Phipps et al.'s study, ${ }^{54}$ which explored a large population-based sample of individuals with CRCs $(n=1,344)$ to determine the relationship between molecular subtypes and survival. In our previous study in which the eight-marker panel was used, CIMP-H had different prognostic implications according to tumor location and was significantly associated with a poor prognosis in rectal cancers but not in proximal or distal colon cancers. ${ }^{16}$ However, when the survival analysis was restricted to stage III or high-risk stage II CRCs treated with adjuvant FL plus oxaliplatin (FOLFOX), CIMP was not statistically significant. ${ }^{17}$ CIMP status did not matter in either the overall survival or recurrence-free survival time in adjuvant FOLFOX-treated stage III or high-risk stage II CRCs. ${ }^{17,55}$ Instead, concurrent methylation of both CDKN2A (p16) and NEUROG1 was significantly associated with shorter diseasefree survival or overall survival time. ${ }^{55}$ Shiovitz et al.'s study ${ }^{56}$ has explored the association of CIMP with response to adjuvant FL versus irinotecan plus FL (IFL) using Laird's five-marker panel and samples $(n=615)$ drawn from a large randomized phase 3 trial (C89803). Shiovitz et al.'s study ${ }^{56}$ demonstrated that patients with stage III, CIMP-H/non-MSI-H colon cancers have longer overall and disease-free survival times when irinotecan is added to adjuvant FL.

\section{CIMP-LOW CRCs}

CRCs with less extensive CIMP-specific methylation were designated as CIMP-L tumors. ${ }^{57,58}$ Tumors are defined as CIMP$\mathrm{L}$ when the presence of methylation in the CIMP panel markers does not reach the cutoff value for CIMP-H. For the five-marker panel, tumors with $1 / 5$ to $2 / 5$ or $3 / 5$ methylated markers were defined as CIMP-L. However, for the eight-marker panel, CIMP$\mathrm{L}$ is defined as the presence of $1 / 8$ to $4 / 8$ or 5/8 methylated markers and the upper limit of the methylated markers differs depending on the CIMP-H cutoff value. Although CIMP-H CRCs are believed to develop along the serrated neoplasia pathway, little information is available regarding whether all of the CRCs arising from sessile serrated adenomas or traditional serrated adenomas are CIMP-H. Some review articles suggest that CRCs arising in sessile or traditional serrated adenomas could be CIMP-H or CIMP-L in two-thirds and one-third of cases, respectively. ${ }^{14,15}$ However, CIMP-L CRCs can develop along the classical adenoma-carcinoma sequence because a considerable por- tion of advanced conventional adenomas or carcinomas arising in conventional adenomas are CIMP-L. ${ }^{59}$ Such a mixture of CIMP-L CRCs with different origins causes the clinicopathological features of CIMP-L tumors to be less distinct compared with CIMP-0 tumors or CIMP-H tumors. However, in the literature, studies have shown that CIMP-L tumors are more commonly associated with the male sex and with KRAS mutation than CIMP-0 or CIMP-H tumors, ${ }^{57}$ and CIMP-L and CIMP-0 cases have different prognoses. ${ }^{60}$ These reports show that CIMP-L and CIMP-0 tumors have different clinicopathologic and molecular features and support the development of reliable consensus criteria of CIMP-L.

\section{PRECURSOR LESIONS IN CIMP-HIGH CRC PATIENTS}

Several studies indicate that sessile serrated adenomas and traditional serrated adenomas are precursor lesions for CIMPH CRCs; (1) CIMP-H is found in traditional serrated adenomas with $B R A F$ mutant and sessile serrated adenomas at a frequency $>50 \%$, whereas CIMP-H is rarely, if ever, found in conventional adenomas; ${ }^{6}$ (2) polyps contiguous with CIMP-H CRCs are sessile serrated adenomas or traditional serrated adenomas; ${ }^{59}$ and (3) BRAF mutations, which are frequently found in CIMP-H CRCs, are detected in sessile serrated adenomas and traditional serrated adenomas at frequencies $>50 \%$ but are rarely found in conventional adenomas. ${ }^{6}$ CIMP-H CRCs may have different precursor lesions depending on MSI status. It is widely accepted that sessile serrated adenomas are the precursor lesion of CIMPH/MSI-H CRCs. ${ }^{9,12}$ Of the two types of traditional serrated adenomas, traditional serrated adenomas arising from pre-existing microvesicular hyperplastic polyps or sessile serrated adenomas are thought to be precursor lesions for CIMP-H CRCs with $B R A F$ mutations. Traditional serrated adenomas arising from goblet cell-rich hyperplastic polyps might be precursor lesions for CIMP-H CRCs with KRAS mutations or CIMP-L CRCs with KRAS mutations. However, because advanced conventional adenomas frequently have CIMP-L and KRAS mutations, CIMP-L CRCs with KRAS mutations that arise from conventional adenomas are thought to be more frequent than those arising from traditional serrated adenomas.

\section{SERRATED ADENOCARCINOMAS VERSUS SERRATED PATHWAY ADENOCARCINOMAS}

Serrated pathway adenocarcinomas refer to CRCs that develop along the serrated neoplasia pathway, while serrated adenocarcinomas refer to a histopathological variant that is defined by histological features, including (1) serrated epithelia, (2) intracellular and extracellular mucin, (3) eosinophilic cytoplasms, (4) abundant cytoplasms, (5) vesicular nuclei, (6) distinct nucleoli, (7) $<10 \%$ necrosis, and (8) papillary fronds and cell balls in the mucin pool. ${ }^{14,61}$ Of these histological features, at least six of the first 
seven are required for the diagnosis of serrated adenocarcinoma. Serrated adenocarcinomas, comprising 7\% to 12\% of CRCs, are more frequent in women, with over half located in the cecum or ascending colon, and one-third located in the rectum. ${ }^{7,62-64}$ Serrated adenocarcinomas have been shown to have worse clinical behavior compared with stage-matched conventional CRCs. ${ }^{65}$ Although serrated adenocarcinomas are a type of serrated pathway adenocarcinomas, all serrated pathway adenocarcinomas are not serrated adenocarcinomas. Because 30\% to 35\% of CRCs are thought to arise through the serrated neoplasia pathway in Western populations, ${ }^{14,58,66}$ approximately one-half to twothirds and one-third of serrated pathway adenocarcinomas are estimated to show CIMP-H status and serrated morphology, respectively. ${ }^{7,23,24,62-64,67}$ However, in the literature, there are no available studies in which CIMP status was assessed in serrated adenocarcinomas or serrated morphology was analyzed in CIMP-H CRCs.

Although morphological criteria define serrated adenocarcinomas, the diagnosis of serrated pathway adenocarcinomas remains a problem. Practically, individual serrated adenomas cannot be longitudinally followed up until progression into CRCs. While no longitudinal observations are available, CRCs could be regarded as serrated pathway adenocarcinomas in circumstances in which (1) cancers are in direct contiguity with serrated adenomas, (2) cancers are CIMP-H, or (3) cancers are serrated adenocarcinomas. Of the five molecular subtypes proposed by Jass, three molecular subtypes, including (1) CIMPH/MSI-H, (2) CIMP-H/non-MSI-H, and (3) CIMP-L/non-MSI-H subtypes, can be found in serrated pathway adenocarcinomas, with the first two subtypes and the third type comprising twothirds and one-third of serrated pathway adenocarcinomas, respectively. ${ }^{58,66}$ Serrated pathway adenocarcinomas with the CIMP-L/non-MSI-H subtype are difficult to identify unless these cancers are serrated adenocarcinomas or directly contiguous with serrated adenomas. Because CIMP-L CRCs can arise from either the classical adenoma-carcinoma sequence or the serrated neoplasia pathway, ${ }^{59}$ determination of CIMP status alone cannot differentiate whether CIMP-L CRCs arise from the serrated neoplasia pathway or from the classical adenoma-carcinoma sequence. Auxiliary tools are necessary to identify CIMP-L serrated pathway adenocarcinomas. In a previous study, alterations of immunophenotypical features were found in the serrated neoplasia pathway, including the early gain of gastric differentiation and the late loss of intestinal differentiation. ${ }^{68}$ Gastric type markers, including ANXA10, VSIG1, CTSE, TFF2, MUC5AC, and MUC6, could be utilized in combination to detect CIMP-L serrated pathway CRCs.

In summary, CRCs are heterogeneous diseases in terms of their molecular carcinogenesis pathways and morphological multistep progression routes. CRCs that arise along the serrated neoplasia pathway are histomorphologically and molecularly different from CRCs that develop along the classical adenoma- carcinoma sequence. Associations between CIMP-H CRCs and proximal-colon predilection and high frequencies of MSI-H and BRAF mutations could be inferred from the fact that the premalignant lesions of CIMP-H CRCs are sessile serrated adenomas with high frequencies of BRAF mutations and CIMP$H$. Because two-thirds of serrated pathway adenocarcinomas do not exhibit serrated morphology, CIMP examination and auxiliary immunohistochemical studies should be performed for the identification of serrated pathway adenocarcinomas with no serrated morphology. Although the prognostic value of CIMP status is controversial, individual CIMP panel markers are found to have prognostic values in adjuvant FOLFOX-treated patients with stage III CRC. Future studies are needed to develop immunohistochemical marker panels to identify serrated pathway adenocarcinomas in CIMP-L CRCs.

\section{CONFLICTS OF INTEREST}

No potential conflict of interest relevant to this article was reported.

\section{ACKNOWLEDGEMENTS}

This study was supported by a grant from the Basic Science Research Program through the National Research Foundation (NRF) funded by the Korean Ministry of Education (2013R1A1A2059080), an NRF grant funded by the Korean Ministry of Science, ICT and Future planning (2011-0030049), a grant (2009-0093820) from the Priority Research Centers Program of the NRF, and a grant from the Korea Health Technology R\&D Project of the Korea Health Industry Development Institute funded by the Korean Ministry of Health and Welfare (HI14C1277).

\section{REFERENCES}

1. Keshet I, Schlesinger Y, Farkash S, et al. Evidence for an instructive mechanism of de novo methylation in cancer cells. Nat Genet 2006;38:149-153.

2. Schlesinger Y, Straussman R, Keshet I, et al. Polycomb-mediated methylation on Lys27 of histone $\mathrm{H} 3$ pre-marks genes for de novo methylation in cancer. Nat Genet 2007;39:232-236.

3. Iino H, Jass JR, Simms LA, et al. DNA microsatellite instability in hyperplastic polyps, serrated adenomas, and mixed polyps: a mild mutator pathway for colorectal cancer? J Clin Pathol 1999;52:5-9.

4. Jass JR. Serrated adenoma and colorectal cancer. J Pathol 1999;187:499-502.

5. Jass JR. Serrated route to colorectal cancer: back street or super highway? J Pathol 2001;193:283-285.

6. Bettington ML, Walker NI, Rosty C, et al. A clinicopathological and molecular analysis of 200 traditional serrated adenomas. Mod Pathol 2015;28:414-427. 
7. Mäkinen MJ, George SM, Jernvall P, Mäkelä J, Vihko P, Karttunen TJ. Colorectal carcinoma associated with serrated adenoma: prevalence, histological features, and prognosis. J Pathol 2001;193:286294.

8. Goldstein NS. Small colonic microsatellite unstable adenocarcinomas and high-grade epithelial dysplasias in sessile serrated adenoma polypectomy specimens: a study of eight cases. Am J Clin Pathol 2006;125:132-145.

9. Jass JR, Young J, Leggett BA. Hyperplastic polyps and DNA microsatellite unstable cancers of the colorectum. Histopathology 2000;37:295-301.

10. Aaltonen LA, Peltomäki P, Mecklin JP, et al. Replication errors in benign and malignant tumors from hereditary nonpolyposis colorectal cancer patients. Cancer Res 1994;54:1645-1648.

11. Jass JR. Colorectal adenomas in surgical specimens from subjects with hereditary non-polyposis colorectal cancer. Histopathology 1995;27:263-267.

12. Jass JR, lino H, Ruszkiewicz A, et al. Neoplastic progression occurs through mutator pathways in hyperplastic polyposis of the colorectum. Gut 2000;47:43-49.

13. Bettington ML, Chetty R. Traditional serrated adenoma: an update. Hum Pathol 2015;46:933-938.

14. Bettington M, Walker N, Clouston A, Brown I, Leggett B, Whitehall V. The serrated pathway to colorectal carcinoma: current concepts and challenges. Histopathology 2013;62:367-386.

15. Young J, Jenkins M, Parry S, et al. Serrated pathway colorectal cancer in the population: genetic consideration. Gut 2007;56:1453-1459.

16. Bae JM, Kim JH, Cho NY, Kim TY, Kang GH. Prognostic implication of the $\mathrm{CpG}$ island methylator phenotype in colorectal cancers depends on tumour location. Br J Cancer 2013;109:1004-1012.

17. Han SW, Lee HJ, Bae JM, et al. Methylation and microsatellite status and recurrence following adjuvant FOLFOX in colorectal cancer. Int J Cancer 2013;132:2209-2216.

18. Slattery ML, Curtin K, Sweeney C, et al. Diet and lifestyle factor associations with $\mathrm{CpG}$ island methylator phenotype and BRAF mutations in colon cancer. Int J Cancer 2007;120:656-663.

19. Carethers JM, Murali B, Yang B, et al. Influence of race on microsatellite instability and CD8+ T cell infiltration in colon cancer. PLoS One 2014;9:e100461.

20. Nosho K, Irahara N, Shima K, et al. Comprehensive biostatistical analysis of $\mathrm{CpG}$ island methylator phenotype in colorectal cancer using a large population-based sample. PLoS One 2008;3:e3698.

21. Ahn JB, Chung WB, Maeda 0, et al. DNA methylation predicts recurrence from resected stage III proximal colon cancer. Cancer 2011;117:1847-1854.

22. Yagi K, Akagi K, Hayashi H, et al. Three DNA methylation epigenotypes in human colorectal cancer. Clin Cancer Res 2010;16:2133.

23. Weisenberger DJ, Siegmund KD, Campan M, et al. CpG island methylator phenotype underlies sporadic microsatellite instability and is tightly associated with BRAF mutation in colorectal cancer.
Nat Genet 2006;38:787-793.

24. Ogino S, Kawasaki T, Kirkner GJ, Kraft P, Loda M, Fuchs CS. Evaluation of markers for $\mathrm{CpG}$ island methylator phenotype (CIMP) in colorectal cancer by a large population-based sample. J Mol Diagn 2007;9:305-314.

25. Cerami E, Gao J, Dogrusoz U, et al. The cBio cancer genomics portal: an open platform for exploring multidimensional cancer genomics data. Cancer Discov 2012;2:401-404.

26. Kang GH, Lee S, Kim WH, et al. Epstein-barr virus-positive gastric carcinoma demonstrates frequent aberrant methylation of multiple genes and constitutes $\mathrm{CpG}$ island methylator phenotype-positive gastric carcinoma. Am J Pathol 2002;160:787-794.

27. Fiorina L, Ricotti M, Vanoli A, et al. Systematic analysis of human oncogenic viruses in colon cancer revealed EBV latency in lymphoid infiltrates. Infect Agent Cancer 2014;9:18.

28. Karpinski P, Myszka A, Ramsey D, Kielan W, Sasiadek MM. Detection of viral DNA sequences in sporadic colorectal cancers in relation to $\mathrm{CpG}$ island methylation and methylator phenotype. Tumour Biol 2011;32:653-659.

29. Newcomb PA, Bush AC, Stoner GL, Lampe JW, Potter JD, Bigler J. No evidence of an association of JC virus and colon neoplasia. Cancer Epidemiol Biomarkers Prev 2004;13:662-666.

30. Militello V, Trevisan M, Squarzon L, et al. Investigation on the presence of polyomavirus, herpesvirus, and papillomavirus sequences in colorectal neoplasms and their association with cancer. Int J Cancer 2009;124:2501-2503.

31. Theodoropoulos G, Panoussopoulos D, Papaconstantinou I, et al. Assessment of JC polyoma virus in colon neoplasms. Dis Colon Rectum 2005;48:86-91.

32. Goel A, Li MS, Nagasaka T, et al. Association of JC virus T-antigen expression with the methylator phenotype in sporadic colorectal cancers. Gastroenterology 2006;130:1950-1961.

33. Rad R, Cadiñanos J, Rad L, et al. A genetic progression model of Braf(V600E)-induced intestinal tumorigenesis reveals targets for therapeutic intervention. Cancer Cell 2013;24:15-29.

34. Fang M, Ou J, Hutchinson L, Green MR. The BRAF oncoprotein functions through the transcriptional repressor MAFG to mediate the CpG Island Methylator phenotype. Mol Cell 2014;55:904-915.

35. Stempak JM, Sohn KJ, Chiang EP, Shane B, Kim YI. Cell and stage of transformation-specific effects of folate deficiency on methionine cycle intermediates and DNA methylation in an in vitro model. Carcinogenesis 2005;26:981-990.

36. Kawakami K, Ruszkiewicz A, Bennett G, Moore J, Watanabe G, Iacopetta B. The folate pool in colorectal cancers is associated with DNA hypermethylation and with a polymorphism in methylenetetrahydrofolate reductase. Clin Cancer Res 2003;9(16 Pt 1):58605835.

37. Samowitz WS, Albertsen H, Sweeney C, et al. Association of smoking, CpG island methylator phenotype, and V600E BRAF mutations in colon cancer. J Natl Cancer Inst 2006;98:1731-1738.

38. Weisenberger DJ, Levine AJ, Long TI, et al. Association of the colorectal $\mathrm{CpG}$ island methylator phenotype with molecular fea- 
tures, risk factors, and family history. Cancer Epidemiol Biomarkers Prev 2015;24:512-519.

39. Toyota M, Ahuja N, Ohe-Toyota M, Herman JG, Baylin SB, Issa JP. CpG island methylator phenotype in colorectal cancer. Proc Natl Acad Sci U S A 1999;96:8681-8686.

40. Toyota M, Ohe-Toyota M, Ahuja N, Issa JP. Distinct genetic profiles in colorectal tumors with or without the $\mathrm{CpG}$ island methylator phenotype. Proc Natl Acad Sci U S A 2000;97:710-715.

41. Issa JP. CpG island methylator phenotype in cancer. Nat Rev Cancer 2004;4:988-993.

42. Samowitz WS, Albertsen H, Herrick J, et al. Evaluation of a large, population-based sample supports a $\mathrm{CpG}$ island methylator phenotype in colon cancer. Gastroenterology 2005;129:837-845.

43. Kim JH, Shin SH, Kwon HJ, Cho NY, Kang GH. Prognostic implications of $\mathrm{CpG}$ island hypermethylator phenotype in colorectal cancers. Virchows Arch 2009;455:485-494.

44. Shen L, Toyota M, Kondo Y, et al. Integrated genetic and epigenetic analysis identifies three different subclasses of colon cancer. Proc Natl Acad Sci U S A 2007;104:18654-18659.

45. Nagasaka T, Sasamoto H, Notohara K, et al. Colorectal cancer with mutation in BRAF, KRAS, and wild-type with respect to both oncogenes showing different patterns of DNA methylation. J Clin Oncol 2004;22:4584-4594.

46. Cancer Genome Atlas Network. Comprehensive molecular characterization of human colon and rectal cancer. Nature 2012;487: 330-337.

47. Simons CC, Hughes LA, Smits KM, et al. A novel classification of colorectal tumors based on microsatellite instability, the $\mathrm{CpG}$ island methylator phenotype and chromosomal instability: implications for prognosis. Ann Oncol 2013;24:2048-2056.

48. Van Rijnsoever M, Elsaleh H, Joseph D, McCaul K, Iacopetta B. $\mathrm{CpG}$ island methylator phenotype is an independent predictor of survival benefit from 5-fluorouracil in stage III colorectal cancer. Clin Cancer Res 2003;9:2898-2903.

49. Hawkins N, Norrie M, Cheong K, et al. CpG island methylation in sporadic colorectal cancers and its relationship to microsatellite instability. Gastroenterology 2002;122:1376-1387.

50. Ward RL, Cheong K, Ku SL, Meagher A, O'Connor T, Hawkins NJ. Adverse prognostic effect of methylation in colorectal cancer is reversed by microsatellite instability. J Clin Oncol 2003;21:37293736.

51. Barault L, Charon-Barra C, Jooste V, et al. Hypermethylator phenotype in sporadic colon cancer: study on a population-based series of 582 cases. Cancer Res 2008;68:8541-8546.

52. Ogino S, Nosho K, Kirkner GJ, et al. CpG island methylator phenotype, microsatellite instability, BRAF mutation and clinical outcome in colon cancer. Gut 2009;58:90-96.

53. Dahlin AM, Palmqvist R, Henriksson ML, et al. The role of the $\mathrm{CpG}$ island methylator phenotype in colorectal cancer prognosis depends on microsatellite instability screening status. Clin Cancer
Res 2010;16:1845-1855.

54. Phipps AI, Limburg PJ, Baron JA, et al. Association between molecular subtypes of colorectal cancer and patient survival. Gastroenterology 2015;148:77-87.e2.

55. Lee DW, Han SW, Cha Y, et al. Different prognostic effect of CpG island methylation according to sex in colorectal cancer patients treated with adjuvant FOLFOX. Clin Epigenetics 2015;7:63.

56. Shiovitz S, Bertagnolli MM, Renfro LA, et al. CpG island methylator phenotype is associated with response to adjuvant irinotecanbased therapy for stage III colon cancer. Gastroenterology 2014; 147:637-645.

57. Ogino S, Kawasaki T, Kirkner GJ, Loda M, Fuchs CS. CpG island methylator phenotype-low (CIMP-low) in colorectal cancer: possible associations with male sex and KRAS mutations. J Mol Diagn 2006;8:582-588.

58. Jass JR. Classification of colorectal cancer based on correlation of clinical, morphological and molecular features. Histopathology 2007;50:113-130.

59. Kwon HJ, Kim JH, Bae JM, Cho NY, Kim TY, Kang GH. DNA methylation changes in ex-adenoma carcinoma of the large intestine. Virchows Arch 2010;457:433-441.

60. Zlobec I, Bihl MP, Foerster A, Rufle A, Terracciano L, Lugli A. Stratification and Prognostic Relevance of Jass's Molecular Classification of Colorectal Cancer. Front Oncol 2012;2:7.

61. Torlakovic E, Skovlund E, Snover DC, Torlakovic G, Nesland JM. Morphologic reappraisal of serrated colorectal polyps. Am J Surg Pathol 2003;27:65-81.

62. Mäkinen MJ. Colorectal serrated adenocarcinoma. Histopathology 2007;50:131-150.

63. Tuppurainen K, Mäkinen JM, Junttila 0, et al. Morphology and microsatellite instability in sporadic serrated and non-serrated colorectal cancer. J Pathol 2005;207:285-294.

64. García-Solano J, Pérez-Guillermo M, Conesa-Zamora P, et al. Clinicopathologic study of 85 colorectal serrated adenocarcinomas: further insights into the full recognition of a new subset of colorectal carcinoma. Hum Pathol 2010;41:1359-1368.

65. García-Solano J, Conesa-Zamora P, Trujillo-Santos J, Mäkinen MJ, Pérez-Guillermo M. Tumour budding and other prognostic pathological features at invasive margins in serrated colorectal adenocarcinoma: a comparative study with conventional carcinoma. Histopathology 2011;59:1046-1056.

66. Snover DC. Update on the serrated pathway to colorectal carcinoma. Hum Pathol 2011;42:1-10.

67. Hinoue T, Weisenberger DJ, Lange CP, et al. Genome-scale analysis of aberrant DNA methylation in colorectal cancer. Genome Res 2012;22:271-282.

68. Kim JH, Kim KJ, Rhee YY, et al. Gastric-type expression signature in serrated pathway-associated colorectal tumors. Hum Pathol 2015;46:643-656. 